\title{
Locally applied cilostazol suppresses neointimal hyperplasia by inhibiting tenascin-c synthesis and smooth muscle cell proliferation in free artery grafts
}

\author{
Kazuya Fujinaga, $M D^{a}$ \\ Koji Onoda, MD, PhDa \\ Kiyohito Yamamoto, $\mathrm{MD}^{\mathrm{a}}$ \\ Kyoko Imanaka-Yoshida, MD, PhD ${ }^{\text {b }}$ \\ Motoshi Takao, MD, PhD \\ Takatsugu Shimono, MD, PhD \\ Hideto Shimpo, MD, $\mathrm{PhD}^{\mathrm{a}}$ \\ Toshimichi Yoshida, MD, PhD ${ }^{b}$ \\ Isao Yada, MD, $\mathrm{PhD}^{\mathrm{a}}$
}

\begin{abstract}
From the Department of Thoracic and Cardiovascular Surgery ${ }^{\mathrm{a}}$ and Department of Pathology, ${ }^{\text {b }}$ Mie University School of Medicine, Tsu, Japan. vitro experiment using cultured smooth muscle cells isolated from rat aorta, cilostazol completely suppressed the tenascin-C mRNA expression induced by plateletderived growth factor-BB.
\end{abstract}

This work was supported by a Grant-in-Aid for General Scientific Research from the Japanese Ministry of Education, Science, Sports, and Culture (No. 12877201 and No. 14370409).

Received for publication July 30, 2003; revisions requested Oct 7, 2003; accepted for publication Nov 5, 2003.

Address for reprints: Koji Onoda, MD, $\mathrm{PhD}$, Department of Thoracic and Cardiovascular Surgery, Mie University School of Medicine, 2-174 Edobashi, Tsu, Mie 5148507, Japan (E-mail: k-onoda@clin. medic.mie-u.ac.jp).

J Thorac Cardiovasc Surg 2004;128:357-63 $0022-5223 / \$ 30.00$

Copyright () 2003 by The American Association for Thoracic Surgery

doi:10.1016/j.jtcvs.2003.11.015

Conclusion: A single topical administration of cilostazol may suppress neointimal hyperplasia by inhibiting cell proliferation and tenascin-C synthesis in free artery grafts, presenting the potential for clinical use in vascular surgery.

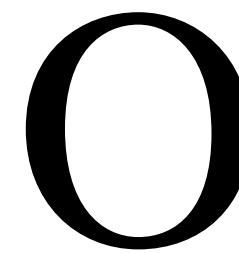

ne of the most serious problems in coronary artery bypass grafting is stenosis at the site of anastomosis, which induces neointimal formation and eventually results in occlusion of the artery graft. Similar to arteriosclerosis, the primary pathophysiologic event associated with neointimal formation is migration of smooth muscle cells (SMCs) from the media to the intima. ${ }^{1,2}$ The SMCs then proliferate and deposit extracellular matrix $(\mathrm{ECM})$, leading to the formation of an occlusive lesion. ${ }^{3-5}$ It has recently been suggested that tenascin-C (TN-C), an ECM protein, may play a crucial role in the remodeling of cardiovascular tissue by affecting cell activity. ${ }^{4-9}$ Marked expression of TN-C has been found in rat balloon-
Objective: Accumulation of smooth muscle cells and extracellular matrix in the intima of artery bypass grafts induces neointimal hyperplasia, resulting in graft failure. We investigated the inhibitory effect of locally applied cilostazol, an inhibitor of cyclic adenosine monophosphate phosphodiesterase III, on neointimal hyperplasia and the role of tenascin- $\mathrm{C}$ synthesis and smooth muscle cell proliferation in free artery grafts.

Methods and Results: We established a distal anastomotic stricture model of free artery graft stenosis using rat abdominal aorta. In this model, neointimal hyperplasia was observed not only in the distal anastomotic site but also in the graft body at postoperative day 14 and was markedly progressed at day 28. Strong expression of tenascin- $\mathrm{C}$ was found in the media and neointima of the graft body. When cilostazol was locally administered around the graft using Pluronic gel, neointimal hyperplasia of the graft was significantly suppressed in comparison with gel-treated control graft. The mean neointima/media area ratio was reduced by $86.6 \%$ for the graft body and by $75.8 \%$ for the distal anastomotic site versus the control. Cilostazol treatment decreased cell proliferation and tenascin-C expression in the neointima. In an in injured artery, ${ }^{10}$ human coronary atherosclerotic plaque, ${ }^{6}$ abdominal aortic aneu- 
$\mathbf{A}$

Examined sections
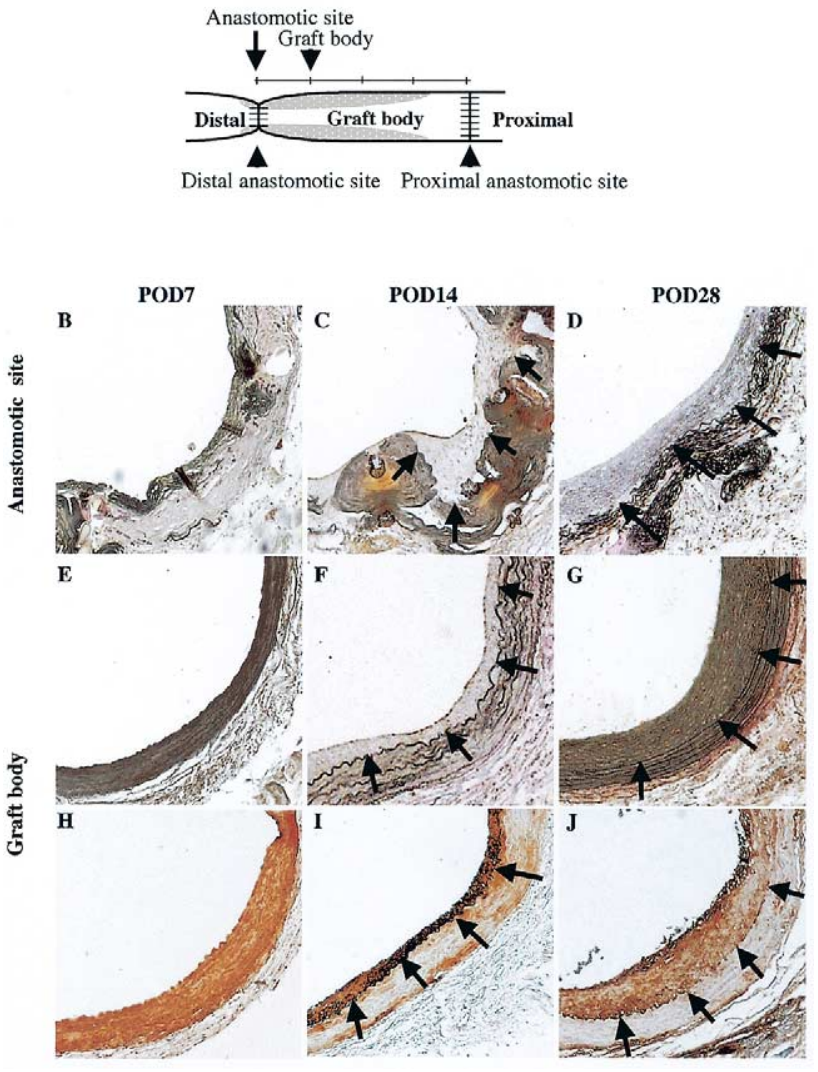

Figure 1. Elastica van Gieson-stained cross sections (B-D and E-F) and immunostaining for TN-C (H-J) of artery grafts (magnification $\times 40)$. A, Diagram of implanted grafts indicating sections examined. B-D, Distal anastomotic stricture site. E-J, Graft body. B, E, H, POD 7. C, F, I, POD 14. D, G, J, POD 28. Arrows indicate internal elastic lamina. POD, Postoperative day.

rysm,,${ }^{11}$ and arterialized human vein grafts, ${ }^{12}$ as well as in the early stage after percutaneous transluminal coronary angioplasty. ${ }^{13}$ In vitro, TN-C blocks adhesion of SMC to fibronectin ${ }^{14}$ and promotes migration. ${ }^{15}$ Platelet-derived growth factor (PDGF) and angiotensin II markedly upregulate the synthesis of TN-C by cultured SMC. ${ }^{14,16}$

A recent report described how locally applied suramin, a growth factor receptor antagonist, inhibited neointimal hyperplasia of mouse vein grafts. ${ }^{17}$ Local administration of phosphodiesterase inhibitors also inhibits neointimal formation in balloon-injured carotid artery by inhibiting medial SMC proliferation. ${ }^{18,19}$ Local application of a drug is an attractive and feasible therapy in bypass surgery with few side effects.

Cilostazol, 6-[4-(1-cyclohexyl-1H-tetrazol-5-yl)butoxy]3,4-dihydro-2(1H)-quinolinone, is a specific inhibitor of cyclic adenosine monophosphate (cAMP) phosphodiesterase III that inhibits platelet aggregation and SMC proliferation and produces vasodilatation. ${ }^{20-22}$ In this study, we investigated whether a single local administration of cilostazol is capable of suppressing neointimal hyperplasia in implanted artery grafts. Furthermore, we also evaluated the effects of cilostazol on TN-C expression and SMC proliferation.

\section{Materials and Methods}

\section{Rat Anastomotic Stricture Model of Free Artery Graft Stenosis}

Male inbred Lewis rats with a mean body weight of $270 \mathrm{~g}$ were used. The animals were handled according to guidelines approved by the Mie University Animal Experiment and Care Committee. The rats were anesthetized by intraperitoneal injection of sodium pentobarbital $(50 \mathrm{mg} / \mathrm{kg})$ and atropine $(0.1 \mathrm{mg})$. Using a sterile technique, we exposed the aorta below the renal artery through an abdominal incision. A $2.0-\mathrm{cm}$ segment of infrarenal aorta was removed from the donor rat. Recipient rats were given an identical abdominal incision. After an injection of $100 \mathrm{U} / \mathrm{kg}$ of heparin into the right lumbar vein, the abdominal aorta was clamped between the renal arteries and the bifurcation of the aorta and then dissected. The donor aortic segment was transplanted into the recipient aorta in an end-to-end fashion. Anastomosis was performed by interrupted 10-0 nylon sutures. In the stricture group, distal anastomotic stricture was experimentally produced by ligating the distal anastomosis with a 22-gauge catheter (outer diameter 0.7 $\mathrm{mm}$ ) using 1-0 silk thread and then removing it. In the control group, the operation was performed without aortic constriction. In the cilostazol-treated group, rats with the anastomotic stricture received $20 \mathrm{mg}$ of cilostazol dissolved in $200 \mu \mathrm{L}$ of dimethyl sulfoxide containing $25 \%$ Pluronic gel locally by topical application around the interposed graft, as previously described. ${ }^{18}$ Rats in the vehicle-treated group received the same dimethyl sulfoxidePluronic gel without cilostazol.

\section{Graft Harvesting and Morphometric Analysis}

Artery grafts were harvested at 7,14 , and 28 days after surgery (6-8 rats per group at each time point) under anesthesia. Recipient rats were perfused with $100 \mathrm{~mL}$ of heparin-prepared saline followed by $100 \mathrm{~mL}$ of $10 \%$ neutral-buffered formalin solution. The grafts were exposed carefully and extracted, including proximal and distal normal aorta, and then postfixed in the same fixative at $4^{\circ} \mathrm{C}$ for 1 to 2 days and embedded in paraffin. For histologic analysis of the graft body, 5 cross sections ( $4 \mu \mathrm{m}$ thick) were cut from the mid-portion between the distal anastomosis and the center of the graft (Figure 1, $A$ ). Four cross sections were selected from the distal anastomotic site (Figure 1, $A$ ) and stained with elastica van Gieson to demarcate the internal elastic lamina. At each section, the ratio between the neointima and the media was calculated using an image analysis system (Adobe Photoshop version 5.0J and NIH Image version 1.61, Macintosh; Adobe Systems, Inc, San Jose, Calif) as previously described. ${ }^{19}$

\section{Immunohistochemical Analysis}

Immunohistochemical staining for TN-C was performed according to the method previously reported. ${ }^{23}$ In brief, the sections were first incubated with mouse monoclonal anti-rat TN-C antibody (1 $\mu \mathrm{g} / \mathrm{mL}$ ) and then with peroxidase-conjugated anti-mouse immu- 
noglobulin G (1:400, MBL, Nagoya, Japan). The sections were treated with diaminobenzidine $/ \mathrm{H}_{2} \mathrm{O}_{2}$ solution and counterstained with hematoxylin.

To identify the cellular source of the neointima, immunohistochemical staining for $\alpha$-smooth muscle actin (SMA) was performed using peroxidase-conjugated anti- $\alpha$-SMA antibody (DAKO, Tokyo, Japan). In addition, to assess the differentiation and proliferation of SMCs, immunohistochemical staining for proliferating cell nuclear antigen (PCNA) was performed using mouse monoclonal antibody against PCNA (PC10, DAKO, Tokyo, Japan), followed by the immunoperoxidase procedure. PCNA index was determined by dividing the number of PCNA-positive cells by the total number of nucleated cells in the neointima and media from each section.

\section{In Situ Hybridization}

TN-C mRNA expression was detected by in situ hybridization at our laboratory using the method previously reported. ${ }^{24}$ Rat aortic tissues were treated with proteinase $\mathrm{K}$ for 10 minutes, and hybridization signals were visualized with alkaline phosphatase-conjugated anti-digoxigenin antibody, followed by incubation in nitrotetrazolium blue/5-bromo-4-chloro-3-indolyl phosphate solution.

\section{Measurement of Tissue and Plasma Concentrations of Cilostazol}

The rats were killed at 14,28 , and 56 days (4 rats at each time point) after local administration of cilostazol to measure plasma and tissue concentrations of the drug. Artery grafts were harvested and washed in cold phosphate-buffered saline to eliminate any possible contamination of drug on the surface. The artery grafts and plasma were stored at $-80^{\circ} \mathrm{C}$ until measurement. Measurement of cilostazol concentrations was performed by high-performance liquid chromatography according to the method previously reported. ${ }^{18}$

\section{Cell Culture}

Rat vascular SMCs were isolated from the thoracic aorta of 10- to 12-week-old male Lewis rats according to the enzyme disperse method previously reported. ${ }^{16}$ Cells were grown in Dulbecco's modified Eagle's medium (Sigma, St Louis, Mo) supplemented with $10 \%$ fetal bovine serum, $100 \mu \mathrm{g} / \mathrm{mL}$ of streptomycin, and 100 $\mathrm{U} / \mathrm{mL}$ of penicillin (Gibco BRL, Life Technologies Inc, Rockville, $\mathrm{Md})$ in a humidified atmosphere $\left(5 \% \mathrm{CO}_{2} / 95 \%\right.$ air $)$ at $37^{\circ} \mathrm{C}$. Cells between passages 4 and 10 were used for all experiments. SMCs were grown to confluence in 6 wells and maintained for 48 hours in a medium containing $0.1 \%$ bovine serum albumin and then for 24 hours in $0.1 \%$ bovine serum albumin alone or medium containing PDGF-BB $(2.5 \mathrm{ng} / \mathrm{mL}$, Sigma) with or without cilostazol (10 $\mu \mathrm{mol} / \mathrm{L})$.

\section{Reverse Transcriptase-Polymerase Chain Reaction Analysis}

Total RNA was isolated from cultured SMCs by the acid guanidine phenol chloroform method. ${ }^{24}$ Total RNA concentrations were determined by spectrophotometry at $260 \mathrm{~nm}$. One $\mu \mathrm{g}$ of total RNA template was converted to cDNA using a first-strand cDNA synthesis kit (Roche, Basel, Switzerland). The forward and reverse primers for TN-C were 5'-GTTTGGAGACCGCAGAGAA-
GAA-3' and 5'-TGTCCCCATATCTTGCCCATCA-3', respectively. The expected size of this specific polymerase chain reaction fragment was 344 base pairs. $\beta$-Actin was used as an internal control, with forward and reverse primers of 5'-GTGGGGCGCCCCAGGCACCA-3' and 5'-CTCCTTAATGTCACGCACGATTTC-3', respectively, and the expected size of the polymerase chain reaction fragment was 540 base pairs. Images were stored, and quantitative analysis was performed using an image analysis system.

\section{Statistical Analysis}

Values were expressed as the mean \pm SD. Statistical analysis between groups was performed by analysis of variance. When a statistically significant overall effect was detected, individual data were compared using the Mann-Whitney test or Bonferroni correction.

\section{Results}

\section{Neointimal Hyperplasia in Rat Artery Grafts}

In the stricture group, neointimal hyperplasia was seen in both the graft body and distal anastomotic site at postoperative day 14 and was markedly progressed at day 28 (Figure 1). Marked neointimal hyperplasia was observed in the graft body close to the distal anastomotic site, gradually decreasing as it extended to the proximal anastomotic site. Therefore, for histologic analysis we selected the midportion between the distal anastomosis and the center of the graft. In the stricture group, the neointima/media area ratios for the graft body and the distal anastomotic site at postoperative day 28 were significantly increased compared with those at day 14 (Figure 2). The ratios for the graft body and the distal anastomotic site at postoperative day 28 in the stricture group were 3 to 4 times higher than those in the nonstricture group (Figure 2). Immunohistochemical staining showed a large number of $\alpha$-SMA-positive cells in the neointima (data not shown).

\section{Expression of TN-C in Rat Artery Grafts}

Immunostaining of TN-C was seen in the media at day 7 after grafting. At days 14 and 28, TN-C staining was detected in the neointima of the graft body, whereas the staining became weaker in the media. TN-C immunolabeling was continuously observed in the adventitia (Figure 1).

In situ hybridization revealed TN-C mRNA-positive cells in the media and adventitia at day 7. At day 14, TN-C-expressing cells were observed predominantly in the neointima (Figure 3). At day 28, the expression of $\mathrm{TN}-\mathrm{C}$ mRNA was no longer seen in either the media or the neointima (data not shown).

\section{Effect of Local Administration of Cilostazol on Neointimal Hyperplasia and TN-C Expression}

In the cilostazol-treated group, neointimal hyperplasia at day 28 was markedly suppressed in both the graft body and the distal anastomotic site compared with the vehicle- 

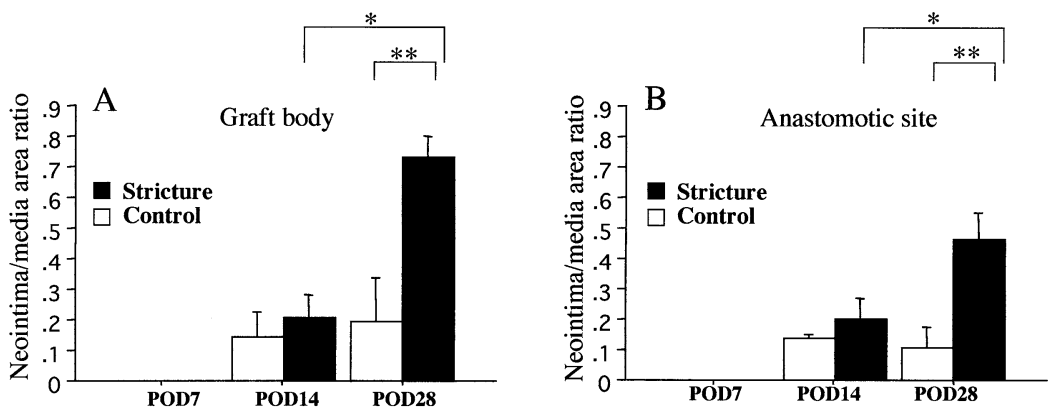

Figure 2. Neointima/media area ratio in graft body (A) and distal anastomotic site (B). Four sections per animal were selected, and data are mean \pm SD of neointima/media area ratio from 5 animals per group at each time point. *Significant difference between PODs 14 and 28 in stricture group (graft body: $\boldsymbol{P}<.001$; anastomotic site: $\boldsymbol{P}<.05$ ). **Significant difference between stricture and non-stricture groups at POD 28 (graft body: $P<.01$; distal anastomotic site: $P<.05)$. POD, Postoperative day.
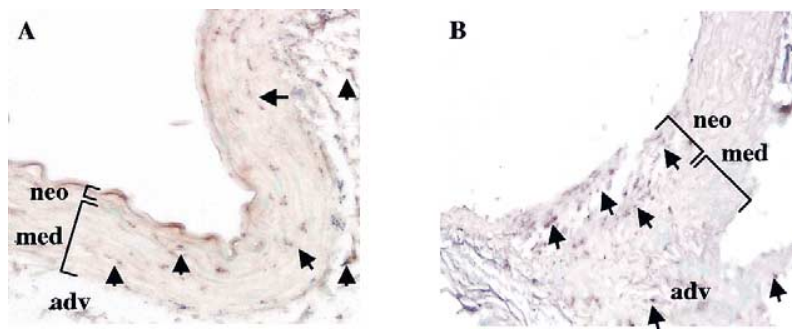

Figure 3. Localization of TN-C by in situ hybridization. TN-C mRNA was detected in the media and adventitia at POD 7 (A), and predominantly in the neointima at day 14 (B). Arrows indicate TN-C mRNA-positive cells. Neo, Neointima; med, media; adv, adventitia.

treated group (Figure 4). Mean neointima/media area ratios in the cilostazol-treated group at day 28 were significantly lower than those in the vehicle-treated group (graft body: $0.055 \pm 0.062$ vs $0.409 \pm 0.172$; anastomotic site: $0.089 \pm$ 0.080 vs $0.368 \pm 0.223, P<.05$, Figure $5, A$ and $B$ ). The rate of reduction compared with the vehicle-treated group was $86.6 \%$ for the graft body and $75.8 \%$ for the anastomotic site. These results indicate that a single local administration of cilostazol significantly suppresses neointimal hyperplasia.

In addition, PCNA indices at day 28 were also significantly lower in the cilostazol-treated group than in the vehicle-treated group (graft body: $3.9 \% \pm 6.1 \%$ vs $37.2 \%$ $\pm 9.5 \%, P<.01$; anastomotic site: $19.2 \% \pm 19.7 \%$ vs $39.7 \% \pm 11.8 \%, P<.05$, Figure $5, C$ and $D$ ). Immunoreactive TN-C in the graft body at day 28 was decreased in the cilostazol-treated group, being observed only in a small portion of the media (Figure 4). These results indicate that local treatment with cilostazol potently inhibits cell proliferation and TN-C expression in the neointima of implanted artery grafts, thus suppressing neointimal hyperplasia.

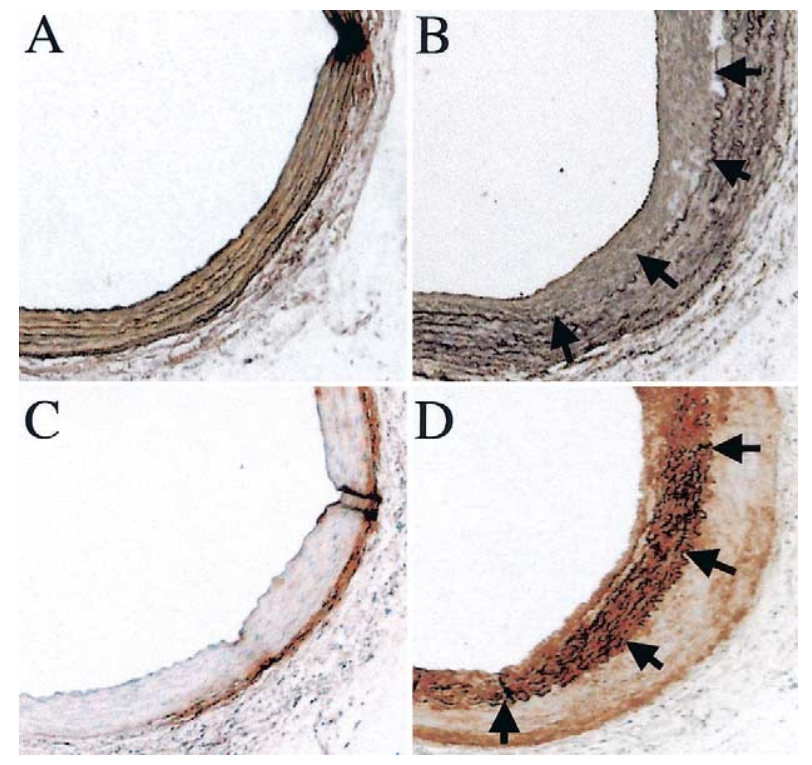

Figure 4. Elastica van Gieson-stained cross sections and immunostaining for TN-C of cilostazol-treated graft bodies at POD 28 (magnification $\times 40$ ). A, C, Cilostazol-treated grafts. B, D, Vehicletreated grafts. A, B, Elastica van Gieson-stained sections; C, D, Immunostaining for TN-C. In the cilostazol-treated graft body, neointimal hyperplasia was markedly inhibited and TN-C-positive staining was observed in only a small portion of the media. Arrows indicate internal elastic lamina.

Tissue and Plasma Concentrations of Cilostazol The plasma concentration of cilostazol was below the detectable level $(<0.01 \mu \mathrm{g} / \mathrm{mL})$ in all samples. In contrast, the mean tissue concentration of cilostazol in the artery graft was $175.44 \mu \mathrm{g} / \mathrm{g}$ tissue at 14 days after topical application. However, the tissue concentration decreased to $0.38 \mu \mathrm{g} / \mathrm{g}$ tissue at day 28 and was below the detectable level at day 56. 
Effect of Cilostazol on TN-C Synthesis in SMCs

To determine whether cilostazol affects TN-C synthesis in SMCs, we investigated TN-C mRNA expression in cultured SMCs isolated from rat aorta. The addition of PDGF-BB increased TN-C mRNA levels 3-fold in comparison with the control. However, the addition of cilostazol $(10 \mu \mathrm{mol} / \mathrm{L})$ almost completely suppressed the up-regulation induced by PDGF-BB (Figure 6).

\section{Discussion}

In this study, we established a new model of free artery graft stenosis in rats based on the premise of free artery grafts in coronary artery bypass grafting. After distal anastomotic stricture, neointimal hyperplasia progressed rapidly in both the graft body and anastomotic site. Furthermore, an increase in PCNA index was also seen in the neointima of the implanted grafts. Distal anastomotic stricture produces blood turbulence or shear stress in grafts, which possibly could induce certain factors for stimulating SMC proliferation and migration and result in neointimal hyperplasia.

Cilostazol is a specific inhibitor of cAMP phosphodiesterase III that is clinically used for the treatment of peripheral arterial occlusive disease by oral delivery. ${ }^{25}$ Cilostazol inhibits PDGF-induced SMC proliferation. ${ }^{21}$ It was reported that systemic administration of cilostazol at $30 \mathrm{mg} / \mathrm{kg}$ by mouth twice per day inhibited neointimal formation in balloon-injured rat carotid artery by $32 \% .{ }^{26}$ In addition, local administration of cilostazol using Pluronic gel maintained a high concentration of the drug at the application site and suppressed neointimal formation through the direct inhibition of SMC proliferation in balloon-injured rat carotid artery. ${ }^{18}$ Therefore, we examined the effect of cilostazol applied locally to implanted artery grafts in suppressing neointimal hyperplasia in our rat model. Topical application of cilostazol to the implanted graft decreased the neointima/ media area ratio to as low as $1 / 7$ in the graft body and to as low as $1 / 4$ at the anastomotic stricture site compared with the vehicle-treated control. PCNA index was also decreased to $1 / 10$ in the graft body and to $1 / 2$ in the stenotic site of the anastomosis. These results indicate that cilostazol inhibits neointimal hyperplasia at least partly by inhibiting cell proliferation in the neointima.

ECM proteins play a crucial role in the remodeling of blood vessels by their effects on cell adhesion, growth, migration, and apoptosis. ${ }^{27} \mathrm{TN}-\mathrm{C}$, an ECM protein, is implicated in the activation of cell migration from the media to the intima in vascular lesions. ${ }^{14}$ In fact, marked TN-C expression is found in the restenotic neointima in the early stage after PTCA, when cells most actively migrate from the media to the neointima. ${ }^{13}$ In the present study, we observed a high level of TN-C expression in the neointima of control grafts but little expression in cilostazol-treated grafts. Sup-
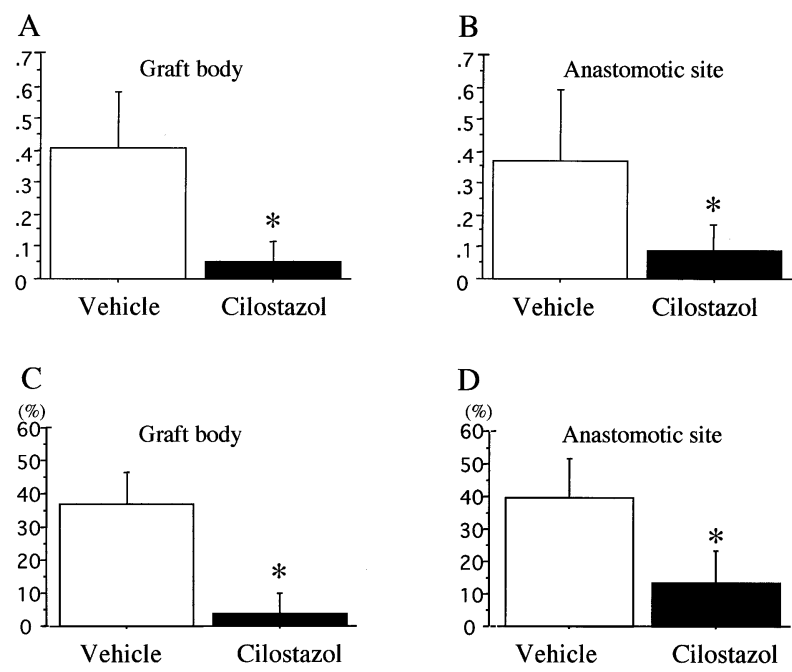

Figure 5. Effect of cilostazol in inhibiting neointimal hyperplasia in artery grafts at POD 28. A, B, Neointima/media area ratio. C, D, PCNA index. A, C, Graft body. B, D, Distal anastomotic site. Four sections per animal were selected, and data are mean \pm SD of neointima/media area ratio and PCNA index from 5 animals per group. Significant differences were seen between the cilostazoltreated and vehicle-treated groups $\left({ }^{*} P<.05\right)$.

pression of TN-C synthesis seems to be another mechanism behind the effect of cilostazol.

The results of previous in vitro studies demonstrated that PDGF-BB is a powerful stimulator of TN-C expression in SMCs. ${ }^{14}$ Therefore, we examined the effects of cilostazol on TN-C expression in cultured SMCs after treatment with PDGF-BB. The increased TN-C mRNA expression induced by PDGF-BB was completely inhibited by the addition of cilostazol.

It has been shown that the cAMP-protein kinase A signal pathway inhibits the transmission of Ras signals from the plasma membrane by preventing the Ras-dependent activation of Raf-1, resulting in inhibition of mitogen-activated protein kinase activation. ${ }^{28-30}$ Mitogen-activated protein kinase phosphorylates a variety of regulatory proteins and transcription factors that play important roles in cell migration, proliferation, and differentiation. ${ }^{31}$ Local administration of 8-bromoadenosine-3',5'-cAMP and phosphodiesterase-inhibitor drugs (aminophylline and amrinone) inhibits neointimal formation in balloon-injured rat carotid artery. ${ }^{19}$ Therefore, the effect of local administration of cilostazol on the inhibition of SMC proliferation and $\mathrm{TN}-\mathrm{C}$ expression could be explained by an increased level of cAMP in SMC.

A recent article demonstrated that adventitial myofibroblasts are the initial source of $\mathrm{TN}-\mathrm{C}$, which stimulates the myofibroblasts themselves to migrate from the adventitia to the intima, and that migration is responsible for the progression of vascular remodeling. ${ }^{32}$ In the present study, using in 
A

\section{$\begin{array}{lll}1 & 2 & 3\end{array}$}
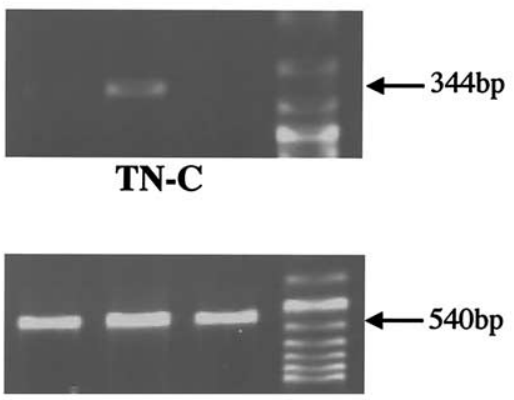

$\beta$-Actin

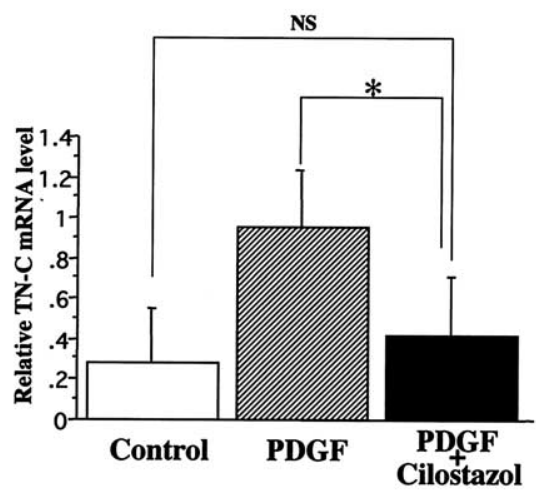

Figure 6. Reverse transcriptase-polymerase chain reaction analysis of TN-C mRNA expression in cultured SMCs isolated from rat aorta. A, Representative reverse transcriptase-polymerase chain reaction data; lane 1: PDGF-BB + cilostazol; lane 2: PDGF-BB; lane 3: no substance added. B, Quantitative analysis of TN-C mRNA levels. *Significant difference between PDGF-BB-treated and PDGF-BB + cilostazol-treated groups $(P<.05)$. TN-C, Tenascin C; $b p$, base pair; PDGF, platelet-derived growth factor; $N S$, not significant.

situ hybridization, we found that $\mathrm{TN}-\mathrm{C}-$ producing cells are initially localized in the adventitia and media but move to the intima during neointimal formation. It seems that the adventitia may be the primary site for vascular remodeling. Therefore, the direct administration of a drug to the adventitia could be dramatically effective in preventing stenosis of free artery grafts. The dramatic inhibitory effect of local administration of cilostazol on intimal hyperplasia can be attributed to the drug's contribution to the adventitial remodeling of artery grafts. In addition, there is the possibility that the other effects of the drug, antithrombotic and vasodilatory effects, contribute to vascular remodeling.

The potential for clinical use of cilostazol on implanted artery grafts in vascular surgery warrants further investigation.

\section{References}

1. Ross R. The pathogenesis of atherosclerosis: a perspective for the 1990s. Nature. 1993;362:801-9.

2. Hosono M, Ueda M, Suehiro S, et al. Neointimal formation at the sites of anastomosis of the internal thoracic artery grafts after coronary artery bypass grafting in human subjects: an immunohistochemical analysis. J Thorac Cardiovasc Surg. 2000;120:319-28.

3. Sottiurai VS, Yao JST, Flinn WR, et al. Intimal hyperplasia and neointima: an ultrastructural analysis of thrombosed grafts in humans. Surgery. 1983;93:809-17.

4. Clowes AW, Gown AM, Hanson SR, et al. Mechanism of arterial graft failure. 1. Role of cellular proliferation in early healing of PTFE prostheses. Am J Pathol. 1985;118:43-54.

5. Ogata T, Kurabayashi M, Hoshino Y, et al. Inducible expression of basic transcription element-binding protein 2 in proliferating smooth muscle cells at the vascular anastomotic stricture. J Thorac Cardiovasc Surg. 2000;119:983-9.

6. Wallner $\mathrm{K}$, Li C, Shah PK, et al. Tenascin-C is expressed in macrophage-rich human coronary atherosclerotic plaque. Circulation. 1999; 99:1284-9.
7. Imanaka-Yoshida K, Hiroe M, Nishikawa T, et al. Tenascin-C modulates adhesion of cardiomyocytes to extracellular matrix during tissue remodeling after myocardial infarction. Lab Invest. 2001;81:1015-24.

8. Imanaka-Yoshida K, Hiroe M, Yasutomi Y, et al. Tenascin-C is a useful marker for disease activity in myocarditis. J Pathol. 2002;197: 388-94.

9. Sato M, Toyozaki T, Odaka K, et al. Detection of experimental autoimmune myocarditis in rats by 111 In monoclonal antibody specific for tenascin-C. Circulation. 2002;106:1397-402.

10. Hedin J, Holm J, Hansson GK. Induction of tenascin in rat arterial injury: relationship to altered smooth muscle cell phenotype. Am J Pathol. 1991;139:649-56.

11. Satta J, Soini Y, Pöllänen R, et al. Tenascin expression is associated with a chronic inflammatory process in abdominal aortic aneurysms. $J$ Vasc Surg. 1997;26:670-5.

12. Wallner K, Li C, Fishbein MC, et al. Arterialization of human vein grafts is associated with tenascin-C expression. $J$ Am Coll Cardiol. 1999;34:871-5.

13. Imanaka-Yoshida K, Matsuura R, Isaka N, et al. Serial extracellular matrix changes in neointimal lesions of human coronary artery after percutaneous transluminal coronary angioplasty: clinical significance of early tenascin-C expression. Virchows Archiv. 2001;439:185-90.

14. LaFleur DW, Fagin JA, Forrester JS, et al. Cloning and characterization of alternatively spliced isoforms of rat tenascin: platelet-derived growth factor-BB markedly stimulates expression of spliced variants of tenascin mRNA in arterial smooth muscle cells. J Biol Chem. 1994;269:20757-63.

15. LaFleur DW, Chiang J, Fagin JA, et al. Aortic smooth muscle cells interact with tenascin-C through its fibrinogen-like domain. $J$ Biol Chem. 1997;272:32798-803.

16. Sharifi BG, LaFleur DW, Pirola CJ, et al. Angiotensin II regulates tenascin gene expression in vascular smooth muscle cells. J Biol Chem. 1992;267:23910-5.

17. Hu Y, Zou Y, Dietrich H, et al. Inhibition of neointima hyperplasia of mouse vein grafts by locally applied suramin. Circulation. 1999;100: 861-8.

18. Ishizaka $\mathrm{N}$, Taguchi $\mathrm{J}$, Kimura $\mathrm{Y}$, et al. Effects of a single local administration of cilostazol on neointimal formation in balloon-injured rat carotid artery. Atherosclerosis. 1999;142:41-6.

19. Indolfi C, Avvedimento EV, Di Lorenzo E, et al. Activation of cAMPPKA signaling in vivo inhibits smooth muscle cell proliferation induced by vascular injury. Nat Med. 1997;3:775-9. 
20. Kimura Y, Tani T, Kanbe T, et al. Effect of cilostazol on platelet aggregation and experimental thrombosis. Arzneimittelforshung. 1985; 35:1144-9.

21. Takahashi S, Oida K, Fujiwara R, et al. Effect of cilostazol, a cyclic AMP phosphodiesterase inhibitor, on the proliferation of rat aortic smooth muscle cells in culture. J Cardiovasc Pharmacol. 1992;20:900-6.

22. Tanaka T, Ishikawa T, Hagiwara M, et al. Effects of cilostazol, a selective cAMP phosphodiesterase inhibitor, on the contraction of vascular smooth muscle. Pharmacology. 1988;36:313-20.

23. Kusagawa H, Onoda K, Namikawa S, et al. Expression and degeneration of tenascin-C in human lung cancers. Br J Cancer. 1998;77:98-102.

24. Noda N, Minoura H, Nishiura R, et al. Expression of tenascin-C in stromal cells of the murine uterus during early pregnancy: induction by interleukin- $1 \alpha$, prostaglandin E2, and prostaglandin F2 $\alpha$. Biol Reprod. 2000;63:1713-20.

25. Hiatt WR. Drug therapy: medical treatment of peripheral arterial disease and claudication. N Engl J Med. 2001;344:1608-21.
26. Inoue $Y$, Kimura $Y$, Hidaka $H$. Role of platelets in vascular intimal hyperplasia. Jpn J Thromb Hemost. 1993;4:297.

27. Ruoslahti E, Vaheri A. Cell-to-cell contact and extracellular matrix. Curr Opin Cell Biol. 1997;9:605-7.

28. Cook S, McCormick F. Inhibition by cAMP of Ras-dependent activation of Raf. Science. 1993;262:1069-72.

29. Sevetson BR, Kong X, Lawrence JC Jr. Increasing cAMP attenuates activation of mitogen-activated protein kinase. Proc Natl Acad Sci U S A. 1993;90:10305-9.

30. Grieco D, Porcellini A, Avvedimento EV, et al. Requirement for cAMP-PKA pathway activation by $M$ phase-promoting factor in the transition from mitosis to interphase. Science. 1996;271:1719-23.

31. Force T, Bonventre JV. Growth factors and mitogen-activated protein kinases. Hypertension. 1998;31:152-61.

32. Wallner K, Sharifi BG, Shah PK, et al. Adventitial remodeling after angioplasty is associated with expression of tenascin mRNA by adventitial myofibroblasts. J Am Coll Cardiol. 2001;37:655-61. 